'Facultad de Medicina, Universidad Finis Terrae. Santiago, Chile. ${ }^{2}$ Centro de Pacientes Críticos, Clínica Las Condes. Santiago, Chile.

${ }^{3}$ Unidad de Respiratorio, Clínica Las Condes. Santiago, Chile.

Recibido el 1 de enero de 2018, aceptado el 5 de julio de 2018 .

Correspondencia a: Dr. Tomás Regueira Clínica Las Condes Estoril 450, Las Condes $+56229485388$ tregueira@clc.cl

\section{Fibrosis pulmonar aguda asociada a intoxicación por Paraquat: caso clínico}

\author{
SEBASTIÁN CARTAGENA ${ }^{1}$, ISRAEL DÍAZ ${ }^{1}$, \\ CONSTANZA GUTIÉRREZ ${ }^{1}$, JORGE DREYSE ${ }^{2,3}$, MATÍAS FLORENZANO ${ }^{3}$, \\ MARCELA PALAVECINO², TOMÁS REGUEIRA ${ }^{2}$
}

\section{Acute pulmonary fibrosis associated with Paraquat poisoning. Report of one case}

Paraquat, a non-selective bipyridyl pesticide, is one of the leading causes of death from intoxication in many parts of Asia and America. It is the second most sold herbicide worldwide, being widely used in Chile. Its ingestion generates toxicity due to the release of superoxide radicals, mainly affecting kidneys, lungs and liver. There is no antidote available. We report a 31 years old male who ingested Paraquat for suicidal purposes. He developed an acute renal and hepatic failure and a rapidly progressive severe respiratory failure with images compatible with acute pulmonary fibrosis. No response to immunosuppressive treatment was observed. He died eight days after admission. The use of cyclophosphamide associated with glucocorticoids could lower risk of death the in these patients, although the pathophysiology of respiratory failure is still under study.

(Rev Med Chile 2018; 146: 938-941)

Key words: Paraquat; Poisoning; Pulmonary Fibrosis.
I a ingestión de paraquat es una de las principales causas de muerte por intoxicación en muchas partes de Asia y países de América y el Pacífico. En Chile no existe epidemiología clara respecto a la intoxicación por paraquat, reportándose entre 4 y 6 casos anuales, estimandose un total de 20-25 casos totales por año. El paraquat es un herbicida bipiridilo no selectivo, corrosivo, de color azul verdoso brillante y olor acre. Es un compuesto seguro de usar en la agricultura, sin embargo, su ingestión tiene una elevada letalidad, razón por la que se ha restringido en muchas partes del mundo. En la actualidad, es el segundo herbicida más vendido a nivel mundial, debido a su rápida acción y bajo costo ${ }^{1}$, de hecho en Chile es ampliamente utilizado.

Gran parte de sus intoxicaciones se deben a ingesta oral, sin embargo, existen reportes descritos en la literatura sobre intoxicación por administración intradérmica e intravenosa ${ }^{2}$. Usualmente los casos reportados de intoxicación en adultos son debido a intento suicida más que exposición accidental, siendo la prevalencia de intentos suicidas mayor en países de $\mathrm{Asia}^{3}$. Su ingestión genera toxicidad por liberación de radicales superóxido, afectando principalmente riñones, pulmones e hígado $^{1,2}$. Sus principales efectos sistémicos son edema pulmonar, convulsiones, falla cardíaca, renal y hepática ${ }^{1}$ y no posee antídoto ${ }^{4}$.

Se presenta a continuación el caso de un paciente con intoxicación por paraquat con fines suicidas, con desenlace fatal.

\section{Caso clínico}

Paciente masculino de 31 años con antecedente de depresión, que consultó por hemorragia digestiva alta $72 \mathrm{~h}$ posterior a ingesta de $30 \mathrm{~mL}$ de paraquat $(138 \mathrm{mg} / \mathrm{kg})$ con intención suicida.

Ingresó consciente, Glasgow 15, con hemodinamia estable y afebril. Al examen físico destaca- 
Fibrosis pulmonar aguda por intoxicación por Paraquat - S. Cartagena et al

Tabla 1. Progresión de parámetros de oxigenación

\begin{tabular}{|lcccc|}
\hline & Día 1 & Día 6 & Día 7 & Día 8 \\
\hline $\mathrm{PaO}_{2}$ & 71 & 61 & 60 & 41 \\
$\mathrm{PaCO}_{2}$ & 34 & 38 & 45 & 96 \\
$\mathrm{pH}$ & 7,34 & 7,45 & 7,43 & 7,11 \\
$\mathrm{PaFi}$ & 337 & 122 & 100 & 40 \\
\hline
\end{tabular}

ban úlceras orales y faríngeas. En su laboratorio de ingreso se evidenció injuria renal aguda (creatinina: 9,58 mg/dL, BUN: $95 \mathrm{mg} / \mathrm{dL}$ ) y hepática (bilirrubina total: 6,3 mg/dL, GOT: $140 \mathrm{U} / \mathrm{L}, \mathrm{GPT}$ $331 \mathrm{U} / \mathrm{L})$. Se realizó tomografía computarizada (TC) de tórax la cual mostró foco de condensa- ción pulmonar compatible con neumonía basal izquierda, sin signos de perforación esofágica. Se inició hidratación parenteral, hemodiálisis, antibióticos empíricos (ceftriaxona) y se ingresó a unidad de cuidados intensivos. Se realizó endoscopía digestiva alta diferida (día 4 de hospitalización) que mostró panesofagitis cáustica grado 2 .

Evolucionó estable hasta el sexto día de hospitalización, presentando insuficiencia respiratoria grave rápidamente progresiva con imágenes compatibles con fibrosis pulmonar aguda (Figura 1). Se inició terapia inmunosupresora con protocolo de metilprednisolona $1 \mathrm{~g} /$ día por 3 días, ciclofosfamida $1 \mathrm{~g}$ /día IV por 2 días y $\mathrm{N}$-acetilcisteína para modular daño por estrés oxidativo, el cual no tuvo respuesta, presentando caída de $\mathrm{PaFi}$ a 40, falleciendo al octavo día de hospitalización.
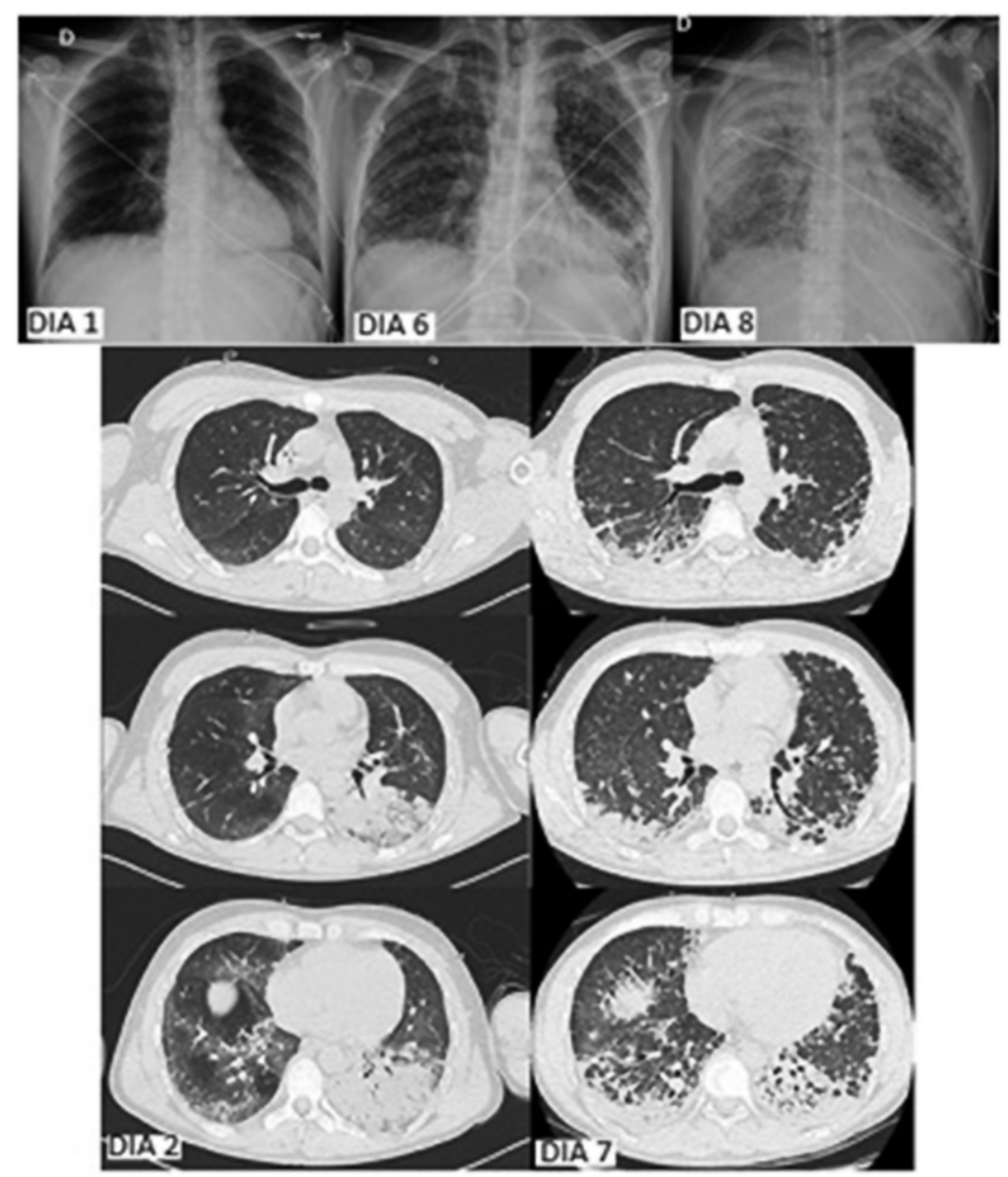

Figura 1. Progresión radiológica del compromiso pulmonar. 


\section{Discusión}

Los herbicidas son ampliamente distribuidos para su uso en agricultura, dentro de ellos el paraquat es uno de los más utilizados ${ }^{5}$. Su uso con fines suicida ha sido reportado en la literatura con pocos registros de sobrevida. Paraquat corresponde a un compuesto bipiridilo altamente polar. Debido a su carácter corrosivo su ingestión provoca lesiones en la mucosa, con edema y ulceraciones dolorosas de boca, faringe, esófago, estómago e intestino. Luego de la ingestión, su absorción oral es rápida e incompleta, alcanzando su máxima concentración plasmática dentro de $2 \mathrm{~h}$. Se distribuye rápidamente en los tejidos, alcanzando su máximo nivel tisular aproximadamente $6 \mathrm{~h}$ tras la ingestión. Los tejidos más afectados son aquellos con alto flujo sanguíneo, particularmente pulmones, riñones, corazón e hígado ${ }^{6}$. Se elimina principalmente por vía renal dentro de las primeras 12 a $24 \mathrm{~h}$, pero en pacientes con intoxicación severa, la vida media de eliminación puede exceder las $100 \mathrm{~h}$.

La toxicidad está dada por su acción sobre el ciclo de reducción y oxidación celular (REDOX), consumiendo NADPH, generando estrés oxidativo con producción de radicales libres, disfunción mitocondrial, peroxidación lipídica y finalmente apoptosis celular, resultando en una intensa respuesta inflamatoria secundaria ${ }^{7}$. En casos severos con deterioro de función renal, se enlentece su eliminación, favoreciendo su concentración tisular y plasmática.

La experiencia clínica sugiere que se desarrolla toxicidad leve con una ingesta de menos de $20 \mathrm{mg} /$ $\mathrm{kg}$, moderada a severa entre $20-40 \mathrm{mg} / \mathrm{kg}$ y fatal con más de $40 \mathrm{mg} / \mathrm{kg}$. En casos de toxicidad moderada pueden observarse náuseas y vómitos, insuficiencia renal, hepática y respiratoria transitoria. La toxicidad grave, puede provocar la muerte en los primeros días debido a perforaciones gastrointestinales o falla multiorgánica. Quienes sobreviven desarrollan fibrosis pulmonar progresiva y mueren durante las siguientes 2 a 4 semanas después de la ingestión.

A nivel pulmonar el paraquat se concentra fundamentalmente por transporte activo, a través del sistema de captación de poliaminas ${ }^{5}$, y su compromiso se caracteriza por injuria pulmonar aguda y fibrosis pulmonar irreversible ${ }^{2}$. El mecanismo de acción exacto de la noxa pulmonar se encuentra en investigación.

El manejo de la toxicidad leve a moderada incluye la descontaminación gastrointestinal con carbón activado, el que tiene un mayor efecto beneficioso antes de $2 \mathrm{~h}$ tras la ingestión, además de descontaminar cualquier otro sitio expuesto (piel u ojos) e inicio de terapia de soporte. No se recomienda el lavado gástrico debido a las lesiones corrosivas producidas por el agente irritante que puede aumentar el riesgo de perforación por el procedimiento. En casos de toxicidad grave, luego de la descontaminación, la reanimación agresiva con fluidos debe tener como objetivo reemplazar las pérdidas y mantener el flujo sanguíneo renal para aumentar la eliminación de toxinas.

La utilización de terapias extracorpóreas de depuración también está descrita, y su mayor beneficio se ha reportado hasta las $4 \mathrm{~h}$ posteriores a la ingestión. Tanto la hemodiálisis como la hemofiltración han resultado efectivas para aumentar la eliminación del paraquat, debido a su bajo porcentaje de unión a proteínas. Posteriormente, en aquellos pacientes que desarrollan falla renal aguda se debe realizar según los criterios estándar.

El tratamiento de soporte incluye monitorización de perfusión, electrolitos plasmáticos, función renal y hepática, y corrección de trastornos asociados según necesidad. La oxigenoterapia no está indicada inicialmente y solo debe aplicarse en caso de hipoxia, debido a que el aporte de oxígeno aumenta la producción de radicales libres y estrés oxidativo.

Finalmente, la terapia inmunosupresora con ciclofosfamida y corticoides se debe considerar, aun cuando la evidencia no es suficiente para realizar una recomendación terapéutica sólida, para tratar el proceso inflamatorio agudo que puede provocar una lesión pulmonar severa y la muerte. El uso de glucocorticoides asociado a terapia inmunosupresora ha sido ampliamente estudiado en pacientes con fibrosis pulmonar inducida por paraquat. Una revisión sistémica realizada por Cochrane mostró que el uso de ciclofosfamida asociado a glucocorticoides tuvo menor riesgo de muerte en este tipo de pacientes ${ }^{8}$. El uso de otras terapias como antioxidantes aún están en estudio, requiriendo mayor evidencia para su uso clínico ${ }^{6}$.

\section{Referencias}

1. Saravu K, Sekhar S, Pai A, Barkur A, Rajesh V, Earla J. Paraquat - A deadly poison: Report of a case and review. Indian J Crit Care Med 2013; 17: 182-4. 
2. Dinis-Oliveira RJ, Duarte JA, Sánchez-Navarro A, Remião F, Bastos ML, Carvalho F. Paraquat poisonings: mechanisms of lung toxicity, clinical features, and treatment. Crit Rev Toxicol 2008; 38 (1): 13-71.

3. Gunnell D, Eddleston M, Phillips MR, Konradsen F. The global distribution of fatal pesticide self-poisoning: systematic review. BMC Public Health 2007; 7: 357.

4. Raghu K, Mahesh V, Sasidhar P, Reddy P, Venkataramaniah V, Agrawal A. Paraquat poisoning: A case report and review of literature. J Family Community Med 2013; 20: 198-200.

5. Moreno M, Salinas R, Troncoso J, Vargas M, Pardo JM.
Intoxicación severa por Paraquat: A propósito de un caso. Revista Chilena de Medicina Intensiva 2016; 31 : 27-30.

6. Gawarammana IB, Buckley NA. Medical management of paraquat ingestion. Br J Clin Pharmacol 2011; 72: 745.

7. Suntres ZE. Role of antioxidants in paraquat toxicity. Toxicology 2002; 180: 65.

8. Li LR, Sydenham E, Chaudhary Bm Beecher D, You C. Glucocorticoid with cyclophosphamide for paraquat-induced lung fibrosis. Cochrane database of systematic reviews 2014, Issue 8, Art. No: CD008084. 